\title{
Sharpenings of Li's criterion for the Riemann Hypothesis
}

\author{
André Voros* \\ CEA, Service de Physique Théorique de Saclay \\ (CNRS URA 2306) \\ F-91191 Gif-sur-Yvette CEDEX (France) \\ E-mail : voros@spht.saclay.cea.fr
}

September 25, 2018

\begin{abstract}
Exact and asymptotic formulae are displayed for the coefficients $\lambda_{n}$ used in Li's criterion for the Riemann Hypothesis. For $n \rightarrow \infty$ we obtain that if (and only if) the Hypothesis is true, $\lambda_{n} \sim n(A \log n+B)$ (with $A>0$ and $B$ explicitly given, also for the case of more general zeta or $L$-functions); whereas in the opposite case, $\lambda_{n}$ has a non-tempered oscillatory form.

Li's criterion for the Riemann Hypothesis $(\mathrm{RH})$ states that the latter is true if and only if a specific real sequence $\left\{\lambda_{n}\right\}_{n=1,2, \ldots}$ has all its terms positive [17, 2]. Here we show that it actually suffices to probe the $\lambda_{n}$ for their large-n behavior, which fully encodes the Riemann Hypothesis by way of a clear-cut and explicit asymptotic alternative. To wit, we first represent $\lambda_{n}$ exactly by a finite oscillatory sum (9), then by a derived integral formula (12), which can finally be evaluated by the saddle-point method in the $n \rightarrow+\infty$ limit. As a result, $\lambda_{n}$ takes one of two sharply distinct and mutually exclusive asymptotic forms: if $\mathrm{RH}$ is true, $\lambda_{n}$ will grow tamely according to (17); if $\mathrm{RH}$ is false, $\lambda_{n}$ will oscillate with an exponentially growing amplitude, in both + and - directions, as described by (18). This dichotomy thus provides a sharp criterion of a new asymptotic type for the Riemann Hypothesis (and for other zeta-type functions as well, replacing (17) by (15)).

This work basically reexposes our results of April 2004 announced in 28, but with an uncompressed text; we also update the references and related comments: for instance, we now derive as (24) a large- $n$ expansion surmised by Maślanka 20] in the meantime.
\end{abstract}

*Also at: Institut de Mathématiques de Jussieu-Chevaleret (CNRS UMR 7586), Université Paris 7 , F-75251 Paris CEDEX 05, France. 


\section{Background and notations.}

We study the sequence [12, 17] (in the notations of Li, whose $\lambda_{n}$ are $n$ times Keiper's)

$$
\lambda_{n}=\sum_{\rho}\left[1-(1-1 / \rho)^{n}\right] \quad(n=1,2, \ldots)
$$

where $\rho$ are the nontrivial zeros of Riemann's $\zeta(s)$, grouped by pairs within summations and products as

$$
\left\{\rho=\frac{1}{2} \pm \mathrm{i} \tau_{k}\right\}_{k=1,2, \ldots,} \quad \text { Re } \tau_{k} \text { positive and non-decreasing; }
$$

we also parametrize each such pair by the single number $x_{k}=\rho(1-\rho)=\frac{1}{4}+\tau_{k}^{2}$.

We will use the completed zeta function $\Xi(s)$ (normalized as $\Xi(0)=\Xi(1)=1$ ) and a symmetrized form of its Hadamard product formula [7, 26],

$$
\Xi(s)=s(s-1) \Gamma(s / 2) \pi^{-s / 2} \zeta(s) \equiv \prod_{k=1}^{\infty}\left[1-\frac{s(1-s)}{x_{k}}\right] ;
$$

we will also use a "secondary" zeta function built over the Riemann zeros,

$$
Z(\sigma)=\sum_{k=1}^{\infty} x_{k}^{-\sigma}, \quad \operatorname{Re} \sigma>\frac{1}{2}
$$

which extends to a meromorphic function in $\mathbb{C}$ having all its poles at the negative halfintegers, plus one pole at $\sigma=+\frac{1}{2}$ [13] of polar part [26]

$$
Z\left(\frac{1}{2}+\varepsilon\right)=R_{-2} \varepsilon^{-2}+R_{-1} \varepsilon^{-1}+O(1)_{\varepsilon \rightarrow 0}
$$

with

$$
R_{-2}=(8 \pi)^{-1}, \quad R_{-1}=-(4 \pi)^{-1} \log 2 \pi \quad \text { in this case. }
$$

Our results 28 mainly relate to those of Keiper [12, of which we only learned later (thanks to K. Maślanka; they were almost never cited), of Bombieri-Lagarias [2] on Li's criterion [17, and of Maślanka [20. Other earlier works considering the $\lambda_{n}$ are [1, 3].

\section{New exact forms for $\lambda_{n}$.}

To reexpress the $\lambda_{n}$, we start from their generating function [12, 17]

$$
f(z)=\frac{\mathrm{d}}{\mathrm{d} z} \log \Xi\left(\frac{1}{1-z}\right) \equiv \sum_{n=1}^{\infty} \lambda_{n} z^{n-1} .
$$

Now the infinite product formula in (3) implies

$$
\log \Xi\left(\frac{1}{1-z}\right)=\sum_{k=1}^{\infty} \log \left[1+\frac{z}{(1-z)^{2} x_{k}}\right]=-\sum_{j=1}^{\infty} \frac{(-1)^{j}}{j}\left[\frac{z}{(1-z)^{2}}\right]^{j} Z(j)
$$


then, expanding $(1-z)^{-2 j}$ by the generalized binomial formula, reordering in powers of $z$ and substituting the output into (7), we get as first result

$$
\lambda_{n}=-n \sum_{j=1}^{n} \frac{(-1)^{j}}{j}\left(\begin{array}{c}
n+j-1 \\
2 j-1
\end{array}\right) Z(j)
$$

Other sums related to the $Z(k)$ are $\mathscr{Z}_{j}=\sum_{\rho} \rho^{-j}$ [22, 16, 26] (often denoted $\sigma_{j}$, but here we use $\sigma$ as variable). It was already known that $\lambda_{n}=\sum_{j=1}^{n}(-1)^{j+1}\left(\begin{array}{c}n \\ j\end{array}\right) \mathscr{Z}_{j}[12$, Equation (27)], and that the $\mathscr{Z}_{j}$ in turn are complicated polynomials in the Stieltjes constants $\left\{\gamma_{k}\right\}_{k<j}$ 22] (for $\lambda_{n}$ and $\gamma_{k}$ see also [21, 3, 4, and references therein). Now the latter relations boil down to $\mathscr{Z}_{j}=1-\left(1-2^{-j}\right) \zeta(j)+(-1)^{j} \eta_{j-1}$ [26. Equation (46)] simply by promoting logarithmic coefficients $\eta_{j}$ [11, 2] (cf. also [16, Equation (12)])

$$
\log [s \zeta(1+s)] \equiv-\sum_{n=1}^{\infty} \eta_{n-1} \frac{s^{n}}{n}
$$

in place of

$$
s \zeta(1+s)=1-\sum_{n=1}^{\infty} \gamma_{n-1} \frac{(-s)^{n}}{(n-1) !} .
$$

The $\lambda_{n}$ thus express as affine combinations of the $\eta_{j}[2$, thm 2].

Remarks:

- the $\eta_{j}$ are the Stieltjes [constants'] cumulants, up to some relabelings [26, 27];

- the $\eta_{j}$ admit an arithmetic expression over the primes [2, Equation (4.1)]; see also [10], which cites [25] for the case $\eta_{0}=-\gamma$;

- relation (9) can be inverted also in closed form, by the same technique as for [26. Equation (48)]:

$$
Z(j)=\sum_{n=1}^{j}(-1)^{n+1}\left(\begin{array}{c}
2 j \\
j-n
\end{array}\right) \lambda_{n}
$$

The expression (9) for $\lambda_{n}$ has some distinctive advantages: it involves the functional equation $\Xi(s)=\Xi(1-s)$ through (3); and unlike the $\mathscr{Z}_{j}$, the $Z(j)$ are positive and gently varying factors: the function $Z(\sigma)$ is regular and very smooth for real $\sigma \geq 1$. Still, (92) is an oscillatory sum, hence difficult to control directly.

Now an integral representation, equivalent to (9) simply by residue calculus, will nevertheless prove much more flexible:

$$
\lambda_{n}=\frac{(-1)^{n} n \mathrm{i}}{\pi} \oint_{C} I(\sigma) \mathrm{d} \sigma, \quad I(\sigma)=\frac{\Gamma(\sigma+n) \Gamma(\sigma-n)}{\Gamma(2 \sigma+1)} Z(\sigma),
$$

where $C$ is a positive contour encircling just the subset of poles $\sigma=+1, \ldots,+n$ of the integrand $I(\sigma)$. 


\section{Asymptotic alternative for $\lambda_{n}, n \rightarrow \infty$.}

The integral formula (12) readily suggests an asymptotic $(n \rightarrow \infty)$ evaluation by the classic saddle-point method [8, using $|I(\sigma)|$ as height function. First, the integration contour $C$ is to be moved in the direction of decreasing $|I(\sigma)|$ as far down as possible: it will thus pass through some saddle-points $\sigma_{*}$ of $|I(\sigma)|$. Then for large $n, I(\sigma)$ peaks near each of these points $\sigma_{*}$, where it makes a contribution of the order of magnitude $\left|I\left(\sigma_{*}\right)\right|$ to the integral: thus the highest saddle-point(s) give(s) the dominant behavior. Consistent asymptotic approximations can also be made inside $I(\sigma)$ throughout: e.g. here, Stirling formulae used for $\Gamma(n+$ const $)$.

This approach, for an integrand not controlled in fully closed form, partly retains an experimental character. We currently advocate it for this problem as a heuristic, rather than rigorous, tool: it predicts the global structure of the results at once, and it treats all the cases readily and correctly, as other techniques confirm.

In the present problem, for large $n$ the landscape of the function $|I(\sigma)|$ is dominantly controlled: by its $\Gamma$ factors, asymptotically $\sim \pi[\sin \pi \sigma \Gamma(2 \sigma+1)]^{-1} n^{2 \sigma-1}$ for finite $\sigma$; and by the polar parts of $I(\sigma)$ near its poles. The induced contour deformation starts as a dilation of $C$ away from the segment $[1, n]$ in all directions, and goes to infinity in the directions $|\arg \sigma|<\frac{\pi}{2}-\delta$. The encountered saddle-points can be of two types here (once $n$ is large enough).

1) For $\sigma$ on the segment $\left(\frac{1}{2}, 1\right),|I(\sigma)| \sim \pi[\sin \pi \sigma \Gamma(2 \sigma+1)]^{-1} n^{2 \sigma-1} Z(\sigma)$ always has one real minimum $\sigma_{\mathrm{r}}(n)$ (tending to $\frac{1}{2}$ as $n \rightarrow \infty$ ), which will be reached by the moving contour; other real saddle-points lie below $\sigma=\frac{1}{2}$ and will not get reached here.

2) Complex saddle-points may enter as well, for which we may focus on the upper halfplane alone: the lower half-plane will give complex-conjugate ("c.c.") contributions. As long as the moving contour stays inside a half-plane $\left\{\operatorname{Re} \sigma>\frac{1}{2}+\varepsilon\right\}$, the integrand can be decomposed as $I=\sum_{k} I_{k}$ according to (4); then for each individual term and within the Stirling approximation for the $\Gamma$-ratio, the saddle-point equation is $0=\frac{\mathrm{d}}{\mathrm{d} \sigma} \log \left|I_{k}(\sigma)\right| \sim$ $\log \left(\sigma^{2}-n^{2}\right)-2 \log 2 \sigma-\log x_{k}$, yielding the saddle-point location

$$
\sigma_{k}(n)=n \mathrm{i} / 2 \tau_{k}
$$

Thus any zero on the critical axis $\left(\tau_{k}\right.$ real) yields a purely imaginary $\sigma_{k}(n)$, not eligible: it lies outside the domain of validity of (4), and its contribution would be subdominant anyway. So in the end, this paragraph excludes the real $\tau_{k}$.

The discussion then fundamentally splits depending on the presence or absence of zeros off the critical axis.

\section{[RH false]}

If there is any zero $\left(\frac{1}{2} \pm \mathrm{i} \tau_{k}\right)$ off the critical axis, we select $\arg \tau_{k}>0$ and assume the case of a simple zero for argument's sake. Paragraph 2) above fully applies to each such zero: the complex saddle-point $\sigma_{k}(n)$ given by (13) lies inside the domain of convergence $\left\{\operatorname{Re} \sigma>\frac{1}{2}\right\}$ as soon as $n>\left|\operatorname{Im} 1 / \tau_{k}\right|^{-1}$, and for $n \rightarrow+\infty$ it gives an additive contribution $\sim\left[\left(\tau_{k}+\mathrm{i} / 2\right) /\left(\tau_{k}-\mathrm{i} / 2\right)\right]^{n}$ (in the usual quadratic approximation of $\log I(\sigma)$ around $\sigma_{k}(n)$ ), 
which grows exponentially in modulus and fluctuates in phase; it will indeed exponentially dominate the contribution of the real saddle-point $\sigma_{\mathrm{r}}(n)$, to be computed later.

This result can also be confirmed rigorously and more directly: by a conformal mapping [2], the function $f(z)$ in (17) has precisely the points $z_{k}=\left(\tau_{k}-\mathrm{i} / 2\right)\left(\tau_{k}+\mathrm{i} / 2\right)^{-1}$ and $z_{k}^{*}$ as simple poles of residue 1 in the unit disk; then a general Darboux theorem 6, chap. VII $\S 2]$ applies here to the poles with $\left|z_{k}\right|<1$, implying that the Taylor coefficients of $f$ (namely, the $\lambda_{n}$ ) indeed have the asymptotic form

$$
\lambda_{n} \sim \sum_{\left\{\left|z_{k}\right|<1\right\}} z_{k}^{-n}+\text { c.c. } \quad\left(\bmod o\left(\mathrm{e}^{\varepsilon n}\right) \forall \varepsilon>0\right), \quad n \rightarrow \infty,
$$

which now holds for multiple zeros as well, counted with their multiplicities. Concretely, $\lambda_{n}$ then oscillates between exponentially growing values of both signs.

Infinitely many zeros $\left(\frac{1}{2} \pm \mathrm{i} \tau_{k}\right)$ off the critical axis are perfectly admissible here: their $z_{k}$ satisfy $\left|z_{k}\right|<1, z_{k} \rightarrow 1$, and the corresponding infinite sums $\sum_{k} z_{k}^{-n}$ still define valid $n \rightarrow+\infty$ asymptotic expansions. On the other hand, the general Darboux formula (14) hopelessly breaks down if the infinitely many $z_{k}^{-n}$ have identical and dominant modulus, which is precisely realized in the case [RH true], with all the $z_{k}$ on the unit circle!

\section{[RH true]}

Here, Darboux's theorem only tells that $\lambda_{n}=o\left(\mathrm{e}^{\varepsilon n}\right) \forall \varepsilon>0$, but it fails to give any clue as to an explicit asymptotic equivalent for $\lambda_{n}$. By contrast, the saddle-point treatment of the integral (12) itself remains thoroughly applicable. Simply now, all the $\tau_{k}$ are real, $Z(\sigma)=O\left(Z(\operatorname{Re} \sigma)|\operatorname{Im} \sigma|^{-3 / 2}\right)$ in $\left\{\operatorname{Re} \sigma>\frac{1}{2}\right\}$, and the contour $C$ can be freely moved towards the boundary $\left\{\operatorname{Re} \sigma=\frac{1}{2}\right\}$ without meeting any of the $\sigma_{k}(n)$ (all of which are purely imaginary). Hence the only dominant saddle-point is now $\sigma_{\mathrm{r}}(n) \in\left(\frac{1}{2}, 1\right)$; it is shaped by the double pole of $Z(\sigma)$ at $\frac{1}{2}$ (itself generated by the totality of Riemann zeros), so that $\sigma_{\mathrm{r}}(n) \sim \frac{1}{2}+\frac{1}{\log n}$. This saddle-point is however non-isolated (it tends to the pole), so the standard saddle-point evaluation using the quadratic approximation of $\log I(\sigma)$ around $\sigma_{\mathrm{r}}(n)$ works very poorly. Here, it is at once simpler and more accurate to keep on deforming a portion of the contour $C$ nearest to $\sigma=\frac{1}{2}$ until it fully encircles this pole (now clockwise), and to note that the ensuing modifications to the integral are asymptotically smaller. Hence for [RH true], $\lambda_{n}$ is given $(\bmod o(n))$ by [28]

$$
\begin{aligned}
\lambda_{n} \sim(-1)^{n} 2 n \operatorname{Res}_{\sigma=1 / 2} I(\sigma) & =2 \pi n\left[2 R_{-2}\left(\psi\left(\frac{1}{2}+n\right)-1+\gamma\right)+R_{-1}\right] \\
& =2 \pi n\left[2 R_{-2}(\log n-1+\gamma)+R_{-1}\right]+O(1 / n)
\end{aligned}
$$

(with $\psi \equiv \Gamma^{\prime} / \Gamma$ estimated by the Stirling formula, $\gamma=$ Euler's constant, and using the polar structure (5) for $Z(\sigma))$.

Prior to using (6) to fix the $R_{-j}$, the argument covers zeros $\rho$ of a more general (arithmetic) Dirichlet series $L(s)$ : as long as the latter has a meromorphic structure and functional equation similar enough to $\zeta(s)$, its secondary zeta function $Z(\sigma)$ keeps a double pole at $\sigma=\frac{1}{2}$ [27]. A related but more concrete requirement can be put on the function 
$N(T)$, the number of zeros of $L(s)$ with $0<\operatorname{Im} \rho<T$ : we ask that for some constants $R_{-2}, R_{-1}$ and some $\alpha<1$ (all now depending on the chosen $L$-series),

$$
N(T)=2 T\left[2 R_{-2}(\log T-1)+R_{-1}\right]+\delta N(T), \quad \delta N(T)=O\left(T^{\alpha}\right) \text { for } T \rightarrow+\infty
$$

(implying $R_{-2} \geq 0$ ). If both conditions (15) and (16) hold, then the polar coefficients of $Z(\sigma)$ in (5) have to be precisely the $R_{-j}$ from (16). All of that is realized in many cases including, but not limited to, Dedekind zeta functions [15, 13, 27] and some Dirichlet $L$-functions [5, 27, 18; see also [14] (discussed at end); in all those instances, $\delta N(T)=$ $O(\log T)$. For the corresponding $\lambda_{n}$, our saddle-point evaluation then always yields: either (16) $\Rightarrow$ (15) if all the zeros have $\operatorname{Re} \rho=\frac{1}{2}-$ or the immediately general result (14) otherwise.

Like (14) before, (15) can be derived quite rigorously but by still another method, previously unknown to us, and written for the Riemann zeros by J. Oesterlé [23] (private communication). We thank him for allowing us to repeat his argument here; we actually word it in the more general present setting (and slightly streamline it). When all the zeros lie on the critical line, first transform the summation (11) into a Stieltjes integral (where $\theta(T)=2 \arctan (1 / 2 T)$ ): $\lambda_{n}=2 \int_{0}^{\infty}[1-\cos n \theta(T)] \mathrm{d} N(T)$, then integrate by parts: $n^{-1} \lambda_{n}=2 \int_{0}^{\pi} \sin n \theta N\left(\frac{1}{2} \cot \frac{\theta}{2}\right) \mathrm{d} \theta$. Now replace $N(T)$ by its large- $T$ form (16) neglecting $\delta N(T)$ and other $O\left(\theta^{-\alpha}\right)$ terms: the error is o(1) by the RiemannLebesgue lemma, mainly because (16) makes $\delta N\left(\frac{1}{2} \cot \frac{\theta}{2}\right)$ integrable over the closed interval $[0, \pi]$; hence $n^{-1} \lambda_{n}=\int_{0}^{\pi} \sin n \theta \frac{1}{\theta}\left[8 R_{-2}\left(\log \frac{1}{\theta}-1\right)+4 R_{-1}\right] \mathrm{d} \theta+o(1)$. Next, change variable: $n \theta=t$, then replace upper $t$-bound $n \pi$ by $+\infty$ again with an $o(1)$ error; thus, $\lambda_{n}=n \int_{0}^{\infty} \frac{\sin t}{t}\left[8 R_{-2}\left(\log \frac{n}{t}-1\right)+4 R_{-1}\right] \mathrm{d} t \quad(\bmod o(n))$, and the last integral evaluates in closed form [9] to yield (15). Unfortunately, we do not see how to extend this purely real-analytic argument to include the [RH false] case as well.

\section{Recapitulation}

As we ended up with two mutually exclusive large- $n$ behaviors for the $\lambda_{n}$, (14) and (15), together they provide a sharp equivalence result. For the Riemann zeros, using the explicit values (6):

Theorem (asymptotic criterion for the Riemann Hypothesis). For $n \rightarrow+\infty$, the sequence $\lambda_{n}$ built over the Riemann zeros follows one of these asymptotic behaviors:

- [RH true $] \quad \Leftrightarrow \quad$ tempered growth to $+\infty$, as

$$
\lambda_{n} \sim \frac{1}{2} n(\log n-1+\gamma-\log 2 \pi) \quad(\bmod o(n))
$$

- [RH false $] \quad \Leftrightarrow \quad$ non-tempered oscillations, as

$$
\lambda_{n} \sim \sum_{\left\{\arg \tau_{k}>0\right\}}\left(\frac{\tau_{k}+\mathrm{i} / 2}{\tau_{k}-\mathrm{i} / 2}\right)^{n}+\text { c.c. } \quad\left(\bmod o\left(\mathrm{e}^{\varepsilon n}\right) \forall \varepsilon>0\right) .
$$

This comprehensive asymptotic statement [28 is new on the [RH false] side to our knowledge, and it also completes some earlier results in the [RH true] case. 
Our main end formula ((17), assuming RH) had actually been displayed by Keiper [12, Equation (37)], but under a somewhat misleading context: in his words, (17) required not just RH but also "very evenly distributed" zeros, and was "much stronger than" RH; no details or proofs were ever supplied. All that hardly points toward our present conclusion that (17) and RH are strictly equivalent.

Oesterlé had a proof of the statement $[\mathrm{RH}$ true $] \Rightarrow$ (17) (see above), but he neither published nor even posted his typescript [23].

In [2, Cor. 1(c)], rather weak exponential lower bounds $\lambda_{n} \geq-c \mathrm{e}^{\varepsilon n}$ were shown to imply $\mathrm{RH}$; the backward assertion [RH true] $\Leftarrow$ (17) is thus also implied by [2] (but cannot be inferred therefrom, as [2] never alludes to asymptotics regarding the $\lambda_{n}$ ).

Our saddle-point approach also handles both cases (17)-(18) at once for the first time.

Numerical data 12, 20] agree well with (17) for $n<7000$ (and even better in the mean if we add the contribution like (15) but from the next pole of $I(\sigma)$,

$$
\delta \lambda_{n}=(-1)^{n} 2 n \operatorname{Res}_{\sigma=0} I(\sigma)=2 Z(0)=+7 / 4
$$

26. Equation (41)], although this correction should not count asymptotically, dominated as it seems by oscillatory terms). Yet the above numerical agreement is inconclusive regarding the Riemann Hypothesis: any currently possible violation of $\mathrm{RH}$ would yield a deviation from (17) detectable only at much higher $n$ (see end of Sect. 4).

\section{An even more sensitive sequence.}

A slightly stronger difference of behavior follows for the special linear combinations (20) below of the coefficients $\eta_{n}$ themselves (defined by (10) above).

Indeed, the definition $\Xi(s)=\Gamma(s / 2) \pi^{-s / 2} s(s-1) \zeta(s)$ substituted into (7) readily yields a decomposition $\lambda_{n}=S_{n}+\bar{S}_{n}$, where [2, thm 2]

$$
S_{n}=-\sum_{j=1}^{n}\left(\begin{array}{l}
n \\
j
\end{array}\right) \eta_{j-1}
$$

is the contribution of $(s-1) \zeta(s)$, and

$$
\bar{S}_{n}=1-\frac{\log 4 \pi+\gamma}{2} n+\hat{S}_{n}, \quad \text { with } \quad \hat{S}_{n}=\sum_{j=2}^{n}\left(\begin{array}{c}
n \\
j
\end{array}\right)(-1)^{j}\left(1-2^{-j}\right) \zeta(j),
$$

is the contribution of the remaining (more explicit) factor. The large- $n$ behavior of the sum $\hat{S}_{n}$ is also computable, by the same route we followed from (91) to (15) through (12). First,

$$
\hat{S}_{n}=\frac{(-1)^{n} n !}{2 \pi \mathrm{i}} \oint_{C^{\prime}} J(\sigma) \mathrm{d} \sigma, \quad J(\sigma)=\frac{\Gamma(\sigma-n)}{\Gamma(\sigma+1)}\left(1-2^{-\sigma}\right) \zeta(\sigma)
$$

integrated around the poles $\sigma=2, \ldots, n$ of $J(\sigma)$; hence for $n \rightarrow+\infty, \hat{S}_{n}$ is asymptotically

$$
(-1)^{n-1} n ! \operatorname{Res}_{\sigma=1} J(\sigma)=\frac{1}{2} n[\psi(n)+\log 2-1+2 \gamma],
$$


now (mod $o\left(n^{-N}\right) \forall N>0$ ) because $J(\sigma)$ has no further singularities; so that finally, using the Stirling expansion for $\psi(n)$ in terms of the Bernoulli numbers $B_{2 k}$,

$$
\bar{S}_{n} \sim \frac{1}{2} n(\log n-1+\gamma-\log 2 \pi)+\frac{3}{4}-\sum_{k=1}^{\infty} \frac{B_{2 k}}{4 k} n^{1-2 k} \quad(n \rightarrow \infty) \quad \text { unconditionally. }
$$

This at once confirms two empirical conjectures made by Maślanka [20, Equations (2.5), $(2.8)]: \bmod o(n)$, the sequence $\left\{\bar{S}_{n}\right\}$ expresses the "trend" (17) obeyed by the sequence $\left\{\lambda_{n}\right\}$ under [RH true]; and to all orders in $n,\left\{\bar{S}_{n}\right\}$ has the asymptotic expansion (24). But whether RH holds or not it makes sense to withdraw the fixed $\left\{\bar{S}_{n}\right\}$-contribution from the previous formulae (17) and (18), as we did in 28] to find:

$$
S_{n}=o(n)
$$

[RH true]

(a case further discussed in [21, 24, 4]), versus

$$
S_{n} \sim \lambda_{n} \sim \sum_{\left\{\arg \tau_{k}>0\right\}}\left(\frac{\tau_{k}+\mathrm{i} / 2}{\tau_{k}-\mathrm{i} / 2}\right)^{n}+\text { c.c. } \quad\left(\bmod o\left(\mathrm{e}^{\varepsilon n}\right) \forall \varepsilon>0\right) \quad \text { [RH false] }
$$

which gives oscillations that blow up exponentially with $n$.

Still, in absolute size, any contribution from (26) will stay considerably smaller than (25) (the background from the set of real $\tau_{j}$ ) up to $n \approx \min _{\left\{\arg \tau_{k}>0\right\}}\left\{\left|\operatorname{Im} 1 / \tau_{k}\right|^{-1}\right\}$ : i.e., $S_{n}$

can only reliably signal a zero violating RH up to a height $|\operatorname{Im} \rho| \lesssim \sqrt{n / 2}$; so vice-versa, since such zeros are now known to require $|\operatorname{Im} \rho| \gtrsim 10^{9}$, they could only be detected by $S_{n}$ for $n \gtrsim 10^{18}$ (see also [1, 23], [14, p. 5]).

Our asymptotic criteria may thus not surpass others in practical sensitivity, but their sharpness is theoretically interesting. For instance, Li's criterion $\lambda_{n}>0(\forall n)$ is now strengthened in that, beyond a finite $n$-range, it refers to ever larger amplitudes either way, i.e., delicate borderline situations such as $\lambda_{n} \rightarrow 0$ are ruled out.

\section{More general cases: a summary}

For the more general zeta-type functions satisfying (15) and (16), the asymptotic alternative for the associated $\lambda_{n}$ is: either (15) if the generalized Riemann Hypothesis (GRH) holds, or (18) otherwise. In the former case, the connection (16) $\Rightarrow$ (15) often makes the asymptotic form of $\lambda_{n}$ fully explicit without any calculation, and it also ensures $R_{-2} \geq 0$ in line with the corresponding generalized Li's criterion [2, 14, 18, 19].

The $\lambda_{n}$ have also been generalized to $L$-functions defined by Hecke operators for the congruence subgroup $\Gamma_{0}(N)$ [18] [19, specially remark 5.4].

More recently and in a broader setting (the $\lambda_{n}$ for automorphic $L$-functions), Lagarias presented an alternative approach to estimate the $\lambda_{n}$ with greater accuracy, mod $O(\sqrt{n} \log n)$ under GRH [14: in the notations of (201)-(21), he directly proves that $\bar{S}_{n}$ obeys (15) mod $O(1)$ unconditionally (thm 5.1), then that $S_{n}=O(\sqrt{n} \log n)$ under GRH (thm 6.1). We note that his leading $\lambda_{n}$-behavior remains tied to the large- $T$ behavior of a counting function according to the rule (16) $\Rightarrow$ (15) [14, Equations (2.11-12),(1.18),(5.2)]. 


\section{References}

[1] Biane, P., Pitman, J. and Yor, M.: Probability laws related to the Jacobi theta and Riemann zeta functions, Bull. Amer. Math. Soc. 38 (2001), 435-465 [Sect. 2.3].

[2] Bombieri, E. and Lagarias, J.C.: Complements to Li's criterion for the Riemann Hypothesis, J. Number Theory 77 (1999), 274-287.

[3] Coffey, M.W.: Relations and positivity results for the derivatives of the Riemann $\xi$ function, J. Comput. Appl. Math. 166 (2004), 525-534.

[4] Coffey, M.W.: New results concerning power series expansions of the Riemann xi function and the Li/Keiper constants, preprint (Jan. 2005); Toward verification of the Riemann Hypothesis: application of the Li criterion, Math. Phys. Anal. Geom. 8 (2005) 211-255.

[5] Davenport, H.: Multiplicative Number Theory, 3rd ed., revised by H.L. Montgomery, Graduate Texts in Mathematics 74, Springer-Verlag (2000) [chap. 16].

[6] Dingle, R.B.: Asymptotic Expansions: Their Derivation and Interpretation, Academic Press (1973).

[7] Edwards, H.M.: Riemann's Zeta Function, Academic Press (1974) [Sect. 1.10].

[8] Erdélyi, A.: Asymptotic Expansions, Dover (1956) [Sect. 2.5].

[9] Gradshteyn, I.S. and Ryzhik, I.M.: Table of Integrals, series and products, 5th ed., A. Jeffrey ed., Academic Press (1994) [Equations (3.721(1)) p. 444 and (4.421(1)) p. 626].

[10] Hashimoto, Y.: Euler constants of Euler products, J. Ramanujan Math. Soc. 19 (2004), 1-14.

[11] Israilov, M.I.: On the Laurent expansion of the Riemann zeta-function, Proc. Steklov Inst. Math. 4 (1983), 105-112 [Russian: Trudy Mat. Inst. i. Steklova 158 (1981), 98-104].

[12] Keiper, J.B.: Power series expansions of Riemann's $\xi$ function, Math. Comput. 58 (1992), 765-773.

[13] Kurokawa, N.: Parabolic components of zeta functions, Proc. Japan Acad. 64, Ser. A (1988), 21-24; Special values of Selberg zeta functions, in: Algebraic K-theory and algebraic number theory (Proceedings, Honolulu 1987), M.R. Stein and R. Keith Dennis eds., Contemp. Math. 83, Amer. Math. Soc. (1989), pp. 133-149.

[14] Lagarias, J.C.: Li coefficients for automorphic L-functions, Ann. Inst. Fourier, Grenoble (2006, to appear) [math.NT/0404394 v4].

[15] Landau, E.: Einführung in die elementare und analytische Theorie der algebraischen Zahlen und der Ideale, Chelsea, New York (1949) [Satz 173 p. 89].

[16] Lehmer, D.H.: The sum of like powers of the zeros of the Riemann zeta function, Math. Comput. 50 (1988), 265-273.

[17] Li, X.-J.: The positivity of a sequence of numbers and the Riemann Hypothesis, J. Number Theory 65 (1997), 325-333. 
[18] Li, X.-J.: Explicit formulas for Dirichlet and Hecke L-functions, Illinois J. Math. 48 (2004), 491-503.

[19] Li, X.-J.: An arithmetic formula for certain coefficients of the Euler product of Hecke polynomials, J. Number Theory 113 (2005), 175-200.

[20] Maślanka, K.: Effective method of computing Li's coefficients and their properties, Experiment. Math. (to appear) [math.NT/0402168 v5].

[21] Maślanka, K.: An explicit formula relating Stieltjes constants and Li's numbers, preprint [math.NT/0406312 v2].

[22] Matsuoka, Y.: A note on the relation between generalized Euler constants and the zeros of the Riemann zeta function, J. Fac. Educ. Shinshu Univ. 53 (1985), 81-82; A sequence associated with the zeros of the Riemann zeta function, Tsukuba J. Math. 10 (1986), 249-254.

[23] Oesterlé, J.: Régions sans zéros de la fonction zêta de Riemann, typescript (2000, revised 2001, uncirculated).

[24] Smith, W.D.: A "good" problem equivalent to the Riemann hypothesis, e-print on http://www.math.temple.edu/ wds/homepage/works.html (2005 version, unpublished).

[25] de la Vallée Poussin, C.-J.: Recherches analytiques sur la théorie des nombres premiers I, Ann. Soc. Sci. Bruxelles 20 (1896), 183-256 [p. 251].

[26] Voros, A.: Zeta functions for the Riemann zeros, Ann. Inst. Fourier, Grenoble 53 (2003), 665-699; erratum: 54 (2004), 1139.

[27] Voros, A.: Zeta functions over zeros of general zeta and L-functions, in: Zeta Functions, Topology and Quantum Physics (Proceedings, Osaka, March 2003), T. Aoki, S. Kanemitsu, M. Nakahara and Y. Ohno eds., Developments in Mathematics 14, Springer-Verlag (2005), pp. 171-196.

[28] Voros, A.: A sharpening of Li's criterion for the Riemann Hypothesis, preprint (Saclay-T04/040 April 2004, unpublished) [math.NT/0404213 v2]. 